\title{
Period-to-Period Variability of Moderate/Severe Obstructive Sleep Apnoea
}

\author{
Charlotte A. Wigston · John R. Stradling $\cdot$ Chris D. Turnbull
}

Received: March 29, 2021 / Accepted: May 1, 2021 / Published online: May 15, 2021

(c) The Author(s) 2021

\begin{abstract}
Introduction: The severity of obstructive sleep apnoea (OSA) is highly variable on a night-tonight basis. Patients are commonly categorised based on the severity of their OSA, and this is then used to influence management and reimbursement, including continuous positive airway pressure (CPAP). We aimed to establish to what extent the OSA severity category changes during two periods of OSA, based on mean and maximum oxygen desaturation index (ODI).

Methods: Patients with a diagnosis of moderate to severe OSA who had been on CPAP for greater than 1 year were included in this study. Subjects underwent two periods of CPAP withdrawal for four nights each.
\end{abstract}

C. A. Wigston

Department of General Medicine, Fiona Stanley

Hospital, Murdoch, Perth, WA, Australia

C. A. Wigston · J. R. Stradling · C. D. Turnbull ( $\square)$ Department of Respiratory Medicine, Oxford Centre for Respiratory Medicine, Churchill Hospital, Oxford University Hospitals NHS Foundation Trust, Oxford OX3 7LE, UK

e-mail: christopher.turnbull@ouh.nhs.uk

J. R. Stradling · C. D. Turnbull

NIHR Oxford Biomedical Research Centre,

University of Oxford, Oxford, UK
Results: Twenty-five patients completed the study. Based on the mean ODI of the four nights, 14 (56\%) patients changed OSA severity categorisation, with three $(12 \%)$ changing category to mild. Based on the maximum ODI of the four nights, nine (36\%) patients changed OSA severity categorisation, with one (4\%) changing category to mild. One third to a half of patients' OSA severity category changed between the two periods of four night's CPAP withdrawal.

Conclusions: OSA is highly variable on a period-to-period basis as well as on a night-to-night basis. We believe the concept of patients having a definable and 'real' level of OSA severity is therefore flawed. OSA severity should be based mainly on symptoms, as these are the dominant reasons for treatment, and the sleep study should be used qualitatively to ascertain whether respiratory events are the likely cause of the symptoms.

Trial Registration: ISRCTN17987510.

Keywords: Continuous positive airway pressure; Sleep apnoea; Obstructive; Polysomnography 


\section{Key Summary Points}

Obstructive sleep apnoea (OSA) severity is highly variable on a night-to-night basis, with patients frequently changing severity category when repeating single-night sleep studies. This has led to the suggestion of multiple-night sleep studies to better categorise OSA.

We have shown that OSA category changed in over half of patients between two four-night study periods. Multiplenight studies do not necessarily overcome variability in a threshold-based categorisation approach of OSA severity.

We believe the concept of patients having a definable and 'real' level of OSA severity is flawed.

We believe that treatment decisions should therefore be based primarily on a patient's symptoms, as these are the dominant reason for treatment.

\section{DIGITAL FEATURES}

This article is published with digital features, including a summary slide, to facilitate understanding of the article. To view digital features for this article go to https://doi.org/10.6084/ m9.figshare.14519742.

\section{INTRODUCTION}

Obstructive sleep apnoea (OSA) is a common disorder, affecting up to 1 billion people worldwide [1]. OSA causes daytime sleepiness and reduced quality of life [2], and OSA is associated with increased cardiovascular risk [3].

Patients are commonly placed into threshold-based categories to guide management; for example, in some countries a certain severity is required to receive reimbursement for continuous positive airway pressure (CPAP) treatment
[4], and recent European directives imply driving restrictions should depend on reaching certain thresholds [5].

However, the associations between the severity of OSA measured on single-night studies and symptoms are weak $[6,7]$. This may be due to night-to-night variability in the severity of OSA $[8,9]$. Multiple-night sleep studies have been suggested as a way of obtaining a more accurate assessment of OSA severity, but the optimum number of sequential studies is not known [8].

We had the opportunity to use data from a previously published study to ascertain whether the categorisation into severity categories could still change between two separate four-night studies [10]. Any categorisation differences between these periods would of course be due to both the known night-to-night variability plus any change in severity over a longer period. We explored differences using both the mean and the maximum OSA severity, as clinical centres use both metrics from multiple-night sleep studies to inform treatment decisions.

\section{METHODS}

The authors had the opportunity to use data from a previous study 'SOX (Supplemental Oxygen during CPAP Withdrawal) trial', which was a single-tertiary-centre, double-blind, crossover trial with randomised treatment order. It was prospectively registered (ISRCTN 17987510) and approved by the South Central Oxford B Research Ethics Committee (REC Reference $15 / \mathrm{SC} / 0007)$. The study was performed in accordance with the Helsinki Declaration of 1964 and its later amendments. All subjects provided informed consent to participate in the study. No patient identifying information is included.

\section{Study Subjects}

Patients included had a diagnosis of moderate to severe OSA (original diagnostic oxygen desaturation index (ODI) $>20 / \mathrm{h}$ ) and had been on CPAP for greater than 1 year, with CPAP compliance of $>4 \mathrm{~h} /$ night. The study subjects 
formed part of a previously published randomised crossover CPAP withdrawal trial, supplemental oxygen versus air [10].

\section{Study Design}

The main study objective was to establish whether patients' OSA severity category changes between the first and second CPAP withdrawal periods based on both the mean and the maximum ODI from the four nights of withdrawal.

All patients underwent an initial four-night screening trial off CPAP and were included in the study if their oxygen desaturation index $4 \%$ $\left(\mathrm{ODI}_{\geq 4}\right.$ ) was $>20 / \mathrm{h}$ on at least one of the four nights. Following this, patients withdrew from CPAP for 14 nights and were randomised to receive either supplemental oxygen or supplemental air (sham) via nasal cannula or loosefitting mask from a concentrator at a rate of $5 \mathrm{~L} /$ min (NewLife Elite, AirSep). After at least 14 nights back on their normal CPAP treatment, patients crossed over. Data from the four nights of CPAP withdrawal during screening, and from the first four nights during CPAP withdrawal onto air, have been compared in this study (see Fig. 1).

The data from the supplementary oxygen arm of the study could not be used for the purposes of this study, as of course the addition of oxygen greatly reduces the ODI without significantly altering the Apnoea-Hypopnoea Index (AHI) [11]. The time between first screening withdrawal and second sham air withdrawal ranged from 2 to 18 weeks.

Nightly home pulse oximetry was used to measure overnight oxygen saturations (Konica Minolta 300i, Tokyo, Japan). The ODI was calculated from these oximetry data.

\section{Analysis}

The main outcomes measure was the number of patients who changed OSA severity category between the first and second CPAP withdrawal periods, based on both the mean and the maximum ODI from the four nights. OSA severity was defined according to ODI as follows: no

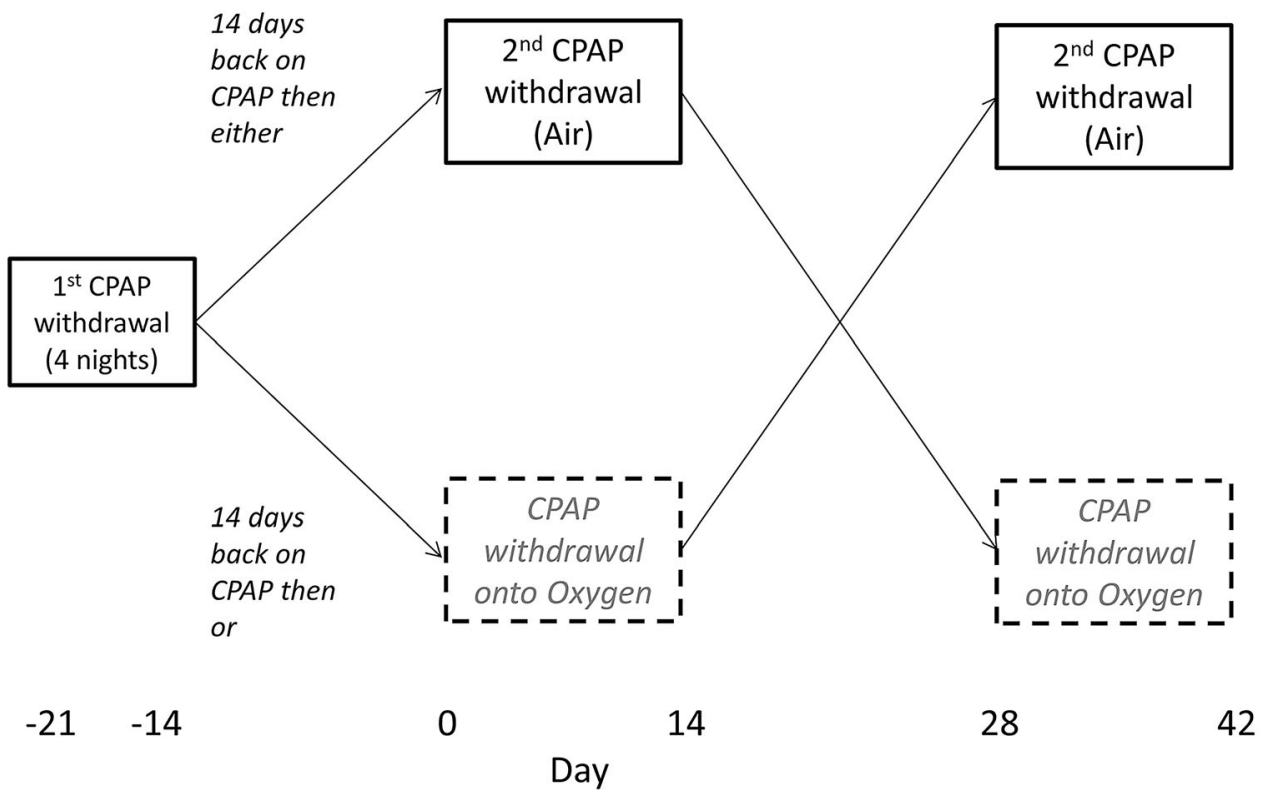

Fig. 1 Study flow diagram 
OSA $<5 / \mathrm{h}$, mild $5-14.9 / \mathrm{h}$, moderate $15-29.9 / \mathrm{h}$, severe $30-59.9 / \mathrm{h}$, and very severe $>60 / \mathrm{h}$.

SPSS version 26.0 (IBM Corp, Armonk, NY, USA) was used for data analysis. Results are shown as mean \pm standard deviation (SD) unless otherwise stated.

\section{RESULTS}

Patients were recruited from June 2015 until June 2017. Details of screening, randomisation, and withdrawals are shown in the CONSORT flow diagram (Fig. 2). Of the initial 57 patients who were enrolled, 25 patients completed the study. Table 1 shows the demographic characteristics of the patients.

The median time from diagnosis to this study ranged from 6.8 years $(100 \%$ range 2.0-19.9). The median (first quartile, third quartile) time between the first and second CPAP withdrawal periods was 10.7 weeks (7.3, 14).

The mean ODI on CPAP was $4.7 \pm 2.9 / \mathrm{h}$, increasing to $31.5 \pm 7.5 / \mathrm{h}$ during the first CPAP withdrawal period, and $32.7 \pm 7.4 / \mathrm{h}$ during the second withdrawal period.

The group mean ODIs on each night of the two withdrawal periods are shown in Table 2 . Using one-way repeated measures analysis of variance (ANOVA), there were no statistically significant differences in ODI values by night on either the first $(F=1.29, d f=3, p=0.29)$ or the second withdrawal period $(F=1.00, d f=3$, $p=0.40$ ). This excluded a first-night effect of stopping CPAP and allowed the use of data from all four nights of CPAP withdrawal for severity categorisation.

The OSA severity categorisation based on both the mean and the maximum ODI during each withdrawal period is shown in Table 3 . Based on the mean ODI, 14 (56\%) patients changed OSA severity categorisation, with three (12\%) changing category to mild (see Fig. 3). Based on the maximum ODI, nine (36\%) patients changed OSA severity categorisation, with one $(4 \%)$ changing category to mild.

\section{DISCUSSION}

Despite using the data from two multiple-night sleep studies, our results show that there are patients who will still cross thresholds even when multiple-night studies are repeated. This was the case either when using the mean, where approximately half of patients changed category; or the maximum ODI, where approximately one third changed category. This further calls into question the use of arbitrary thresholds to decide any aspect of a patient's management, as is common practice at present [12].

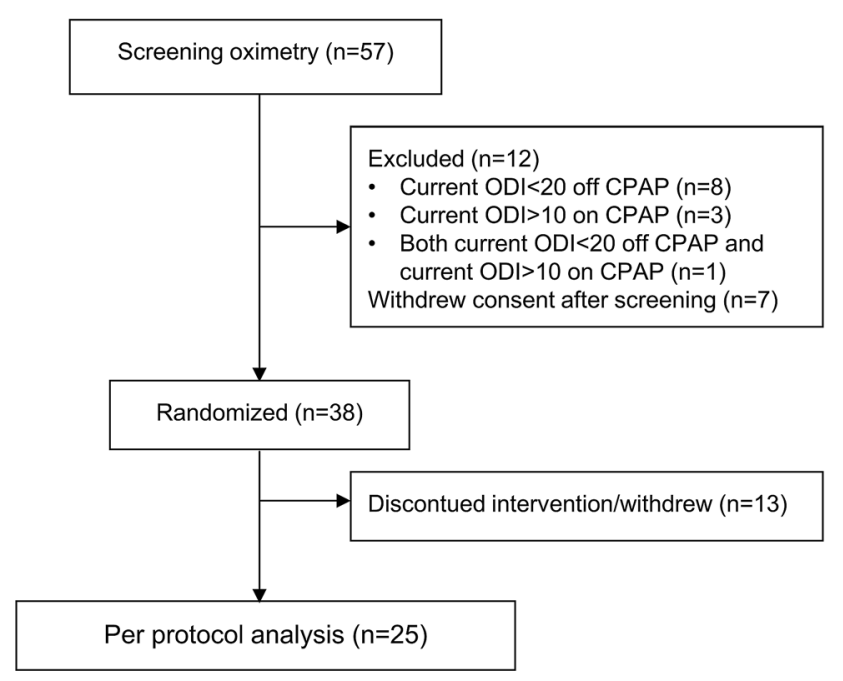

Fig. 2 Details of study screening and enrolment 
Table 1 Demographic characteristics of patients completing the study

\begin{tabular}{ll}
\hline & $\begin{array}{l}\text { All patients } \\
(\boldsymbol{n}=25)\end{array}$ \\
\hline Age, years & $62.7 \pm 6.9$ \\
Male sex & $21(84 \%)$ \\
BMI, km/m ${ }^{2}$ & $35.3 \pm 6.7$ \\
Neck circumference, cm & $48.0(24.3-68.2)$ \\
Mean $\mathrm{ODI}_{\geq 4 \%}$ at diagnosis, per & $50.4 \pm 24.6$ \\
\hline
\end{tabular}

Data shown as mean \pm standard deviation, median (interquartile range) or number (\%)

$B M I$ body mass index, $O D I_{\geq 4 \%}$ oxygen desaturation index of at least $4 \%$

Even amongst patients selected to have moderate OSA on their first CPAP withdrawal, three patients had a mean ODI $<15 / h$ on their second period of CPAP withdrawal, which is below the threshold required for reimbursed CPAP therapy in some countries [4]. As might be expected, using the maximum ODI from these four nights reduced this to only one patient.

Randomised controlled trials have shown CPAP to be effective in reducing symptoms in patients with OSA categorised as mild on the basis of a single-night sleep study [13, 14]. If OSA severity had been measured on multiple nights rather than on a single night in these trials, then some patients with mild OSA might have been re-categorised as moderate OSA, due to regression to the mean of sleep apnoea severity. Furthermore, randomised controlled trials have shown CPAP to be similarly effective across the spectrum of severity of OSA $[13,14]$, and the correlation between OSA severity and sleepiness is weak $[6,7,15]$. We therefore think that the severity of symptoms and likely impact on quality of life are much more important than the severity of OSA on sleep studies when making management decisions.

Table 2 Group mean ODI on nights 1-4 for the first and second withdrawal periods

\begin{tabular}{llllll}
\hline ODI & Night $\mathbf{1}$ & Night 2 & Night 3 & Night $\mathbf{4}$ & $\boldsymbol{p}$ \\
\hline First withdrawal & $30.0 \pm 15.2$ & $32.2 \pm 15.8$ & $30.9 \pm 12.7$ & $36.0 \pm 16.3$ & 0.29 \\
Second withdrawal & $30.9 \pm 13.3$ & $32.2 \pm 16.7$ & $32.6 \pm 18.8$ & $35.4 \pm 16.0$ & 0.40 \\
\hline
\end{tabular}

Data shown as mean \pm standard deviation

Table 3 Patients' OSA severity categorisation on the first and second CPAP withdrawal periods for both the mean and the maximum ODI

\begin{tabular}{lllll}
\hline \multicolumn{2}{l}{$\begin{array}{l}\text { Categorisation based on the mean ODI of the } 4 \\
\text { nights }\end{array}$} & $\begin{array}{l}\text { Categorisation based on the maximum ODI of the } 4 \\
\text { nights }\end{array}$ \\
\cline { 2 - 3 } First withdrawal & Second withdrawal & & First withdrawal & Second withdrawal \\
\hline No OSA & $0(0 \%)$ & $0(0 \%)$ & $0(0 \%)$ & $0(0 \%)$ \\
Mild & $0(0 \%)$ & $3(12 \%)$ & $0(0 \%)$ & $1(4 \%)$ \\
Severe & $11(44 \%)$ & $9(36 \%)$ & $8(32 \%)$ & $4(16 \%)$ \\
Very severe & $1(4 \%)$ & $12(48 \%)$ & $15(60 \%)$ & $17(68 \%)$ \\
\hline
\end{tabular}

Data shown as $n$ (\%)

$O S A$ obstructive sleep apnoea, $C P A P$ continuous positive airway pressure, $O D I$ oxygen desaturation index 


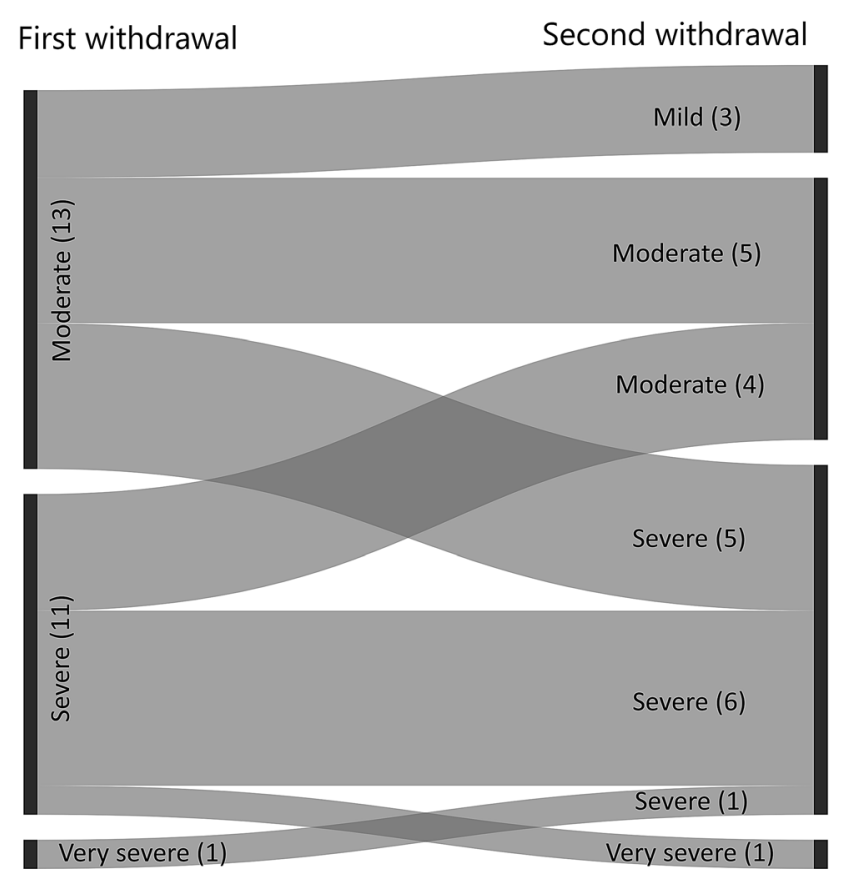

Fig. 3 Sankey plot to show change in patients' OSA severity between first and second CPAP withdrawal periods. OSA obstructive sleep apnoea. OSA severity defined according to patient's oxygen desaturation index;

Threshold-based categorisations of OSA have shown at the epidemiological level that only the more severe OSA tends to be associated with cardiovascular events [3], and therefore might justify continuing this high threshold to guide treatment. However, the SAVE study showed that CPAP does not reduce cardiovascular events [16]; therefore, it still seems reasonable that symptoms should remain the principal reason to treat OSA.

There are limitations of this study. This was an opportunistic study looking at data from a previously published study and therefore is exploratory. Returning OSA was assessed using overnight pulse oximetry and not polysomnography, which is currently the gold standard technique for diagnosing OSA in some countries. Pulse oximetry was chosen for this study as it is more practical to measure this at home over multiple nights. Without using polysomnography, differences in total sleep time, differing sleep architecture, and differing time in a supine position could all account for differences in the ODI, and are in fact the likely no OSA $<5 / \mathrm{h}$, mild $5-14.9 / \mathrm{h}$, moderate $15-29.9 / \mathrm{h}$, severe $30-59.9 / \mathrm{h}$, and very severe $>60 / \mathrm{h}$

explanation for some of the observed night-tonight variation [17]. However, our use of mean values from multiple-night sleep studies should have reduced any such variability. In many sleep centres that use polygraphy, sleep length, sleep state, and posture issues will be part of the variation they observe anyway. Our study only selected patients with an ODI $>20 / \mathrm{h}$ on their first period of CPAP withdrawal; thus the results might have shown regression downwards towards the mean, although the means of the two periods were in fact almost identical. It is possible that patients' weight changed between the first and second withdrawal period, which might have contributed to changes in OSA severity. However, given that the average time between CPAP withdrawal periods was short, and the means of the two periods were almost identical, we feel this is unlikely to explain the changes in OSA severity.

It has been pointed out by others that overnight pulse oximetry in isolation is not recommended for the diagnosis of OSA [18]; however, the correlation between ODI and AHI is very 
high, and our observations are likely to apply to more comprehensive sleep studies. One clear difference between the two periods was that all patients received sham oxygen therapy (air at 5 $\mathrm{L} / \mathrm{min}$ ) during their second CPAP withdrawal, which might have affected sleep. However, if this decreased sleep, we might have expected a different ODI during the second withdrawal period, which was not the case.

\section{CONCLUSIONS}

We have shown that there is a clinically relevant period-to-period variability in OSA severity category. Even using four nights of study, one third to a half of patients' OSA severity category differed between the two periods of CPAP withdrawal. We believe that the concept of a patient having a definable, 'real' and repeatable OSA severity is thus flawed. In our view, assessment of the severity of OSA should be based mainly on symptoms, as these are the dominant reason for treatment and cause of limitations on driving, with the sleep study mainly ascertaining whether respiratory events are likely to be the cause of these symptoms.

\section{ACKNOWLEDGEMENTS}

The authors would like to thank the study participants enrolled in the SOX Trial.

Funding. The original study was supported by the Oxford Radcliffe Hospital Charitable Funds. This research was also supported by the National Institute for Health Research (NIHR) Oxford Biomedical Research Centre. The views expressed are those of the authors and not necessarily of the NHS, the NIHR, or the Department of Health. CT was supported by an NIHR Academic Clinical Lectureship. No funding or sponsorship was received for this study or publication of this article.

Authorship. All named authors meet the International Committee of Medical Journal Editors (ICMJE) criteria for authorship for this article, take responsibility for the integrity of the work as a whole, and have given their approval for this version to be published.

Authorship Contributions. All authors contributed to the concept and design of this study. Charlie Wigston and Chris Turnbull carried out the statistical analyses. Charlie Wigston drafted the first version of the manuscript, and all authors contributed to the final manuscript.

Prior Presentation. The abstract from this study and data were presented at European Respiratory Society Online Congress 2020. The abstract 'Variability of OSA severity classification between two multiple-night sleep studies' was published in European Respiratory Journal 2020, Vol 56 Issue supplement 64.

Disclosures. Charlotte Wigston has nothing to disclose. John Stradling declares personal fees from Bayer and Resmed UK, outside the submitted work. Chris Turnbull declares personal fees from Bayer, outside the submitted work.

Compliance with Ethics Guidelines. The authors had the opportunity to use data from a previous study 'SOX (Supplemental Oxygen during CPAP Withdrawal) trial', which was a single-tertiary-centre, double-blind, crossover trial with randomised treatment order. It was prospectively registered (ISRCTN 17987510) and approved by the South Central Oxford B Research Ethics Committee (REC Reference $15 / \mathrm{SC} / 0007)$. The study was performed in accordance with the Helsinki Declaration of 1964 and its later amendments. All subjects provided informed consent to participate in the study. No patient identifying information is included.

Data Availability. The datasets generated and/or analysed during the current study are available from the corresponding author on reasonable request.

Open Access. This article is licensed under a Creative Commons Attribution-NonCommercial 4.0 International License, which permits any non-commercial use, sharing, adaptation, distribution and reproduction in any medium 
or format, as long as you give appropriate credit to the original author(s) and the source, provide a link to the Creative Commons licence, and indicate if changes were made. The images or other third party material in this article are included in the article's Creative Commons licence, unless indicated otherwise in a credit line to the material. If material is not included in the article's Creative Commons licence and your intended use is not permitted by statutory regulation or exceeds the permitted use, you will need to obtain permission directly from the copyright holder. To view a copy of this licence, visit http://creativecommons.org/licenses/by$\mathrm{nc} / 4.0 /$.

\section{REFERENCES}

1. Benjafield AV, Ayas NT, Eastwood PR, Heinzer R, Ip MSM, Morrell MJ, et al. Estimation of the global prevalence and burden of obstructive sleep apnoea: a literature-based analysis. Lancet Respir Med. 2019;7(8):687-98.

2. Jenkinson C, Davies RJ, Mullins R, Stradling JR. Comparison of therapeutic and subtherapeutic nasal continuous positive airway pressure for obstructive sleep apnoea: a randomised prospective parallel trial. Lancet. 1999;353(9170):2100-5.

3. Marin JM, Carrizo SJ, Vicente E, Agusti AG. Longterm cardiovascular outcomes in men with obstructive sleep apnoea-hypopnoea with or without treatment with continuous positive airway pressure: an observational study. Lancet. 2005;365(9464):1046-53.

4. Fietze I, Penzel T, Alonderis A, Barbe F, Bonsignore MR, Calverly $\mathrm{P}$, et al. Management of obstructive sleep apnea in Europe. Sleep Med. 2011;12(2): 190-7.

5. Bonsignore MR, Randerath $\mathrm{W}$, Riha R, Smyth D, Gratziou C, Goncalves M, et al. New rules on driver licensing for patients with obstructive sleep apnoea: EU Directive 2014/85/EU. Eur Respir J. 2016;47(1): 39-41.

6. Lipford MC, Wahner-Roedler DL, Welsh GA, Mandrekar J, Thapa P, Olson EJ. Correlation of the Epworth Sleepiness Scale and sleep-disordered breathing in men and women. J Clin Sleep Med. 2019;15(1):33-8.
7. Heinzer R, Vat S, Marques-Vidal P, Marti-Soler H, Andries D, Tobback N, et al. Prevalence of sleepdisordered breathing in the general population: the HypnoLaus study. Lancet Respir Med. 2015;3(4): 310-8.

8. Stoberl AS, Schwarz EI, Haile SR, Turnbull CD, Rossi VA, Stradling JR, et al. Night-to-night variability of obstructive sleep apnea. J Sleep Res. 2017;26(6): 782-8.

9. Bittencourt LR, Suchecki D, Tufik S, Peres C, Togeiro $\mathrm{SM}$, Bagnato MC, et al. The variability of the apnoea-hypopnoea index. J Sleep Res. 2001;10(3): 245-51.

10. Turnbull CD, Sen D, Kohler M, Petousi N, Stradling JR. Effect of supplemental oxygen on blood pressure in obstructive sleep apnea (SOX). A randomized continuous positive airway pressure withdrawal trial. Am J Respir Crit Care Med. 2019;199(2):211-9.

11. Mehta V, Vasu TS, Phillips B, Chung F. Obstructive sleep apnea and oxygen therapy: a systematic review of the literature and meta-analysis. J Clin Sleep Med. 2013;9(3):271-9.

12. Epstein LJ, Kristo D, Strollo PJ Jr, Friedman N, Malhotra A, Patil SP, et al. Clinical guideline for the evaluation, management and long-term care of obstructive sleep apnea in adults. J Clin Sleep Med. 2009;5(3):263-76.

13. Wimms AJ, Kelly JL, Turnbull CD, McMillan A, Craig SE, O'Reilly JF, et al. Continuous positive airway pressure versus standard care for the treatment of people with mild obstructive sleep apnoea (MERGE): a multicentre, randomised controlled trial. Lancet Respir Med. 2019;8:349-58.

14. Craig SE, Kohler M, Nicoll D, Bratton DJ, Nunn A, Davies R, et al. Continuous positive airway pressure improves sleepiness but not calculated vascular risk in patients with minimally symptomatic obstructive sleep apnoea: the MOSAIC randomised controlled trial. Thorax. 2012;67(12):1090-6.

15. Siccoli MM, Pepperell JC, Kohler M, Craig SE, Davies RJ, Stradling JR. Effects of continuous positive airway pressure on quality of life in patients with moderate to severe obstructive sleep apnea: data from a randomized controlled trial. Sleep. 2008;31(11):1551-8.

16. McEvoy RD, Antic NA, Heeley E, Luo Y, Ou Q, Zhang $\mathrm{X}$, et al. CPAP for prevention of cardiovascular events in obstructive sleep apnea. N Engl J Med. 2016;375(10):919-31.

17. Joosten SA, O'Driscoll DM, Berger PJ, Hamilton GS. Supine position related obstructive sleep apnea in 
adults: pathogenesis and treatment. Sleep Med Rev. 2014;18(1):7-17.
18. van Zeller M, Drummond M. CPAP holiday: are we there yet? Eur Respir J. 2015;45(2):574-5. 